Communication

\title{
Auxiliary Optomechanical Tools for 3D Cell Manipulation
}

\author{
Ivan Shishkin 1,2,3,*®e , Hen Markovich 2,3, Yael Roichman ${ }^{3,4,5}$ and Pavel Ginzburg 2,3 \\ 1 Faculty of Physics and Engineering, ITMO University, Lomonosova 9, 191002 St. Petersburg, Russia \\ 2 School of Electrical Engineering, Tel Aviv University, Tel Aviv 69978, Israel; markohen@gmail.com (H.M.); \\ pginzburg@post.tau.ac.il (P.G.) \\ 3 Light-Matter Interaction Centre, Tel Aviv University, Tel Aviv 69978, Israel; roichman@tauex.tau.ac.il \\ 4 School of Chemistry, Tel Aviv University, Tel Aviv 69978, Israel \\ 5 School of Physics \& Astronomy, Tel Aviv University, Tel Aviv 69978, Israel \\ * Correspondence: i.shishkin@metalab.ifmo.ru
}

Received: 7 November 2019; Accepted: 9 January 2020; Published: 13 January 2020

check for updates

\begin{abstract}
Advances in laser and optoelectronic technologies have brought the general concept of optomechanical manipulation to the level of standard biophysical tools, paving the way towards controlled experiments and measurements of tiny mechanical forces. Recent developments in direct laser writing (DLW) have enabled the realization of new types of micron-scale optomechanical tools, capable of performing designated functions. Here we further develop the concept of DLW-fabricated optomechanically-driven tools and demonstrate full-3D manipulation capabilities over biological objects. In particular, we resolved the long-standing problem of out-of-plane rotation in a pure liquid, which was demonstrated on a living cell, clamped between a pair of forks, designed for efficient manipulation with holographic optical tweezers. The demonstrated concept paves the way for the realization of flexible tools for performing on-demand functions over biological objects, such as cell tomography and surgery to name just few.
\end{abstract}

Keywords: holographic optical trapping; direct laser writing

\section{Introduction}

Three-dimensional optical microscopy techniques are invaluable tools in modern biomedical and biophysical studies. Imaging techniques include, for example, confocal [1], multiphoton [2], and super-resolution [3] microscopy. In most cases, these methods require the immobilization of objects under study, at least during image acquisition. A common way to obtain three-dimensional (3D) characterization of the investigated objects is to combine fluorescent dyes and image sectioning. This requires additional sample preparation and, in some cases, may harm or alter the studied system. In biomedical diagnostics, it is especially beneficial to obtain such characterization in a label free manner. For this reason, various 3D tomography techniques, based on quantitative phase microscopy, were developed in the past few years. For example, single-cell optical coherence tomography can be implemented [4], however, imaging-based techniques appear more practical. These techniques require the ability to rotate an object in suspension in a controlled manner. Sample scanning can be achieved by several methods, including loading objects under study in gel-filled microcapillaries and rotating them mechanically [5,6]. Optical tweezers also have been demonstrated as a viable tool for object scanning by using single-beam time-shared trap [7] (suitable only for elongated objects like E. coli), and by using multiple optical traps applied to non-spherical objects like diatoms [8] and yeast cells [9].

The possibility of the rotation of an individual cell was demonstrated using a pair of counterpropagating beams from single-mode fibers inserted in the microfluidic channel [10-12], 
using holographic optical trap [13] or electrorotation [14]. However, it should be noted that trapping of living tissue with counterpropagating laser beams or with structured light in holographic tweezers is constrained by localized heating [15] and phototoxicity [16,17].

Our approach provides capabilities of full 3D manipulation of biological samples within solutions and allows achieving a set of essential functionalities, including (i) prevention of photoinduced damage to living cells, (ii) manipulation of transparent/low contrast objects, (iii) 3D manipulation, including rotation, of spherical species. Furthermore, from the fundamental standpoint, we demonstrate the utilization of radiation pressure forces for achieving the controllable rotation of objects. Our general concept is depicted in Figure 1a, where optomechanically driven 'cell clamps' immobilize a biological cell. Those micron-size clamps are fabricated with the help of direct laser writing [18] (DLW). This technique is based on two-photon absorption [19] in photopolymerizable materials [20], which is an extremely viable method for the fabrication of structures with a sub-micron-scale resolution. A few notable examples of DLW-based structures for opto-fluidic applications include force and topography-sensing optically driven scanning microprobes [21-23], light-actuated microsyringes [24], and platforms for targeted light delivery to microscopic objects like cells [25]. It should be noted that several designs were developed earlier to achieve out-of-plane rotation, like paddlewheel [26] and crankshaft-like structure [27], however none of them have been tested for the manipulation of individual cells.

Our auxiliary structures are driven into motion with the help of holographic optical tweezers. Each clamp is illuminated with three beams-a pair for immobilization and the third one for achieving the rotation of the trapped cell (revolver geometry). Those micro-tools allow for the clamping of an object, translating it towards the analyzing apparatus, rotating it, and finally releasing it back to the suspension. Furthermore, the immobilized cell is not directly illuminated by intense laser light and the whole scheme does not rely on cell's parameters, which makes this approach quite universal. We report on the design, fabrication, and use of 3D printed unique cell clamps that enable trapping, translation, and rotation of cells using optical forces focused away from the cell.

\section{Materials and Methods}

The proposed approach towards cell rotation is schematically depicted in Figure 1a. A pair of auxiliary tools fabricated by DLW is detached from substrate and immobilized with trapping laser beams. Afterwards the desired cell is located and is immobilized by a pair of tools driven into its proximity simultaneously.

A scanning electron microscopy (SEM) image of the designed cell clamps, shaped like forks, is presented in Figure 1b,c. The structure has several essential elements: (i) a fork end for clamping a cell; (ii) base and top spheres for optical trapping with gradient forces, which are used to control their position and orientation. The radius of the sphere is large enough compared to the fork core to ensure localized trapping [23]. (iii) Three spheres, forming a revolver operating like a windmill, are placed in between these two spheres. The distance between the centers of 'actuating' spheres and the axis of the symmetry of the structure was set $5 \mu \mathrm{m}$. The clamps are $30 \mu \mathrm{m}$ long and each spherical feature is $5 \mu \mathrm{m}$ in diameter. The distance between the base and top spheres used for immobilization of the tool was chosen to be $18 \mu \mathrm{m}$. It should be noted, that the dimensions of the microtool could be reduced roughly by a factor of 2, however a larger size was chosen for better mechanical stability and to allow easier detachment with a micromanipulator. Before performing optical experiments, the coverslips with the microforks were cured overnight with a UV lamp in order to suppress residual fluorescence from the photoinitiator and to increase their mechanical stiffness.

The projection of a defocused trap on the 'revolver' part of the structure is the optimal way to use radiation pressure to rotate the fork around its axis. Rotation can be initiated by turning the third optical trap on, stopped by turning it off, and reversed by projecting the trap of the other side of the fork in real time using a holographic optical tweezers (HOTs) setup [28]. The setup used green $532 \mathrm{~nm}$ laser, a reflective spatial light modulator (SLM) module, beam expanders and inverted bright field 
microscope. The laser beam was expanded in order to overfill SLM aperture. The SLM is placed in the focus of shrinking telescope, which forms $4 \mathrm{f}$-system with the microscope objective. The zero order spot was blocked in the focal plane of the negative beam expander after the SLM. The beam is reflected upward inside the microscope using a beam splitter cube and is focused using a 100x Olympus oil immersion objective $(\mathrm{NA}=1.4)$ into the sample chamber. The phase masks of the SLM for the trapping and manipulating (open, close and rotate) of the micro-tools were designed using MATLAB (Version R2013b, MathWorks, Inc., Natick, MA, USA) and calculated using Gerchberg-Saxton iterative algorithm. The traps placement has been designed to be symmetric with respect to the zero-order beam to reduce the intensity of the higher diffraction orders of the SLM.

Phosphate buffer saline (PBS) with $0.5 \%$ TWEEN 20 was used as working medium. The structures were mechanically detached from the coverslip using a glass microneedle connected to the micromanipulator (Scientifica Patchstar, Scientifica, East Sussex, UK) before conducting the experiments. After detaching two structures from the surface, the shutter of the laser was opened. By using the motorized stage the structures are brought into the trapping focal spots of the laser and are immobilized. Since the access for the glass capillary was needed, the samples were not sealed in double-glass chamber and the occasional addition of water was needed in order to compensate for the evaporation.

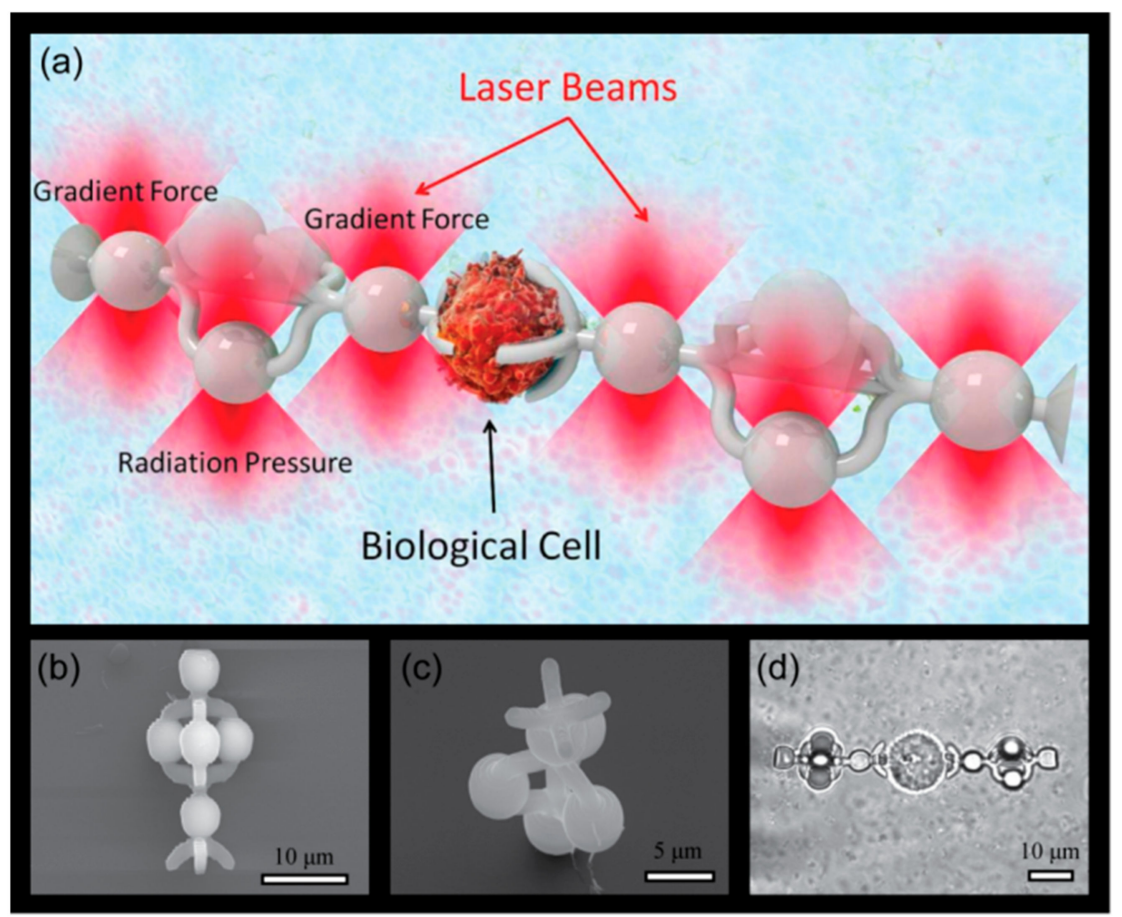

Figure 1. (a) An artist's view of the cell clamps at work. The fork-like shaped clamps are optically trapped in 3D by holographic optical tweezers. Scanning electron microscopy (SEM) side view (b) and top view (c) of fabricated auxiliary microtools. (d) Microscope image of microtools engaged in immobilization of sw480 adenocarcinoma cell.

\section{Results}

In order to demonstrate feasibility of the proposed approach towards object manipulation, it was necessary to show the capability of rotation of the individual micro-tool. After detachment from the coverslip, the tool was immobilized using two generated traps, positioned at anchoring points marked with red crosses in the first frame in Figure 2. After successful immobilization, the third 'actuator' trap was generated the microns off-plane in the spot marked with the cross. The stable trapping of individual tool was achieved with laser power of $0.8 \mathrm{~W}$ incident on SLM, which was distributed between three trapping spots. The relative power of the 'actuator' trap was reduced compared to the two main immobilization traps by $50 \%$ to improve stability of trapping. 
The video sequence of the experiment with a single micro-tool is presented in Figure 2 as set of individual frames captured with $1.5 \mathrm{~s}$ interval (see Supplementary Video S1 for complete sequence). It can be clearly seen that, with the proposed configuration of the traps, the desired axial rotation can be achieved. The rotational motion was induced with the radiation pressure force that pushes one of the beads in the revolver part. The photon momentum is transformed to the structure owing to light absorption in the polymer, which arises from residual molecules of photoinitiator and intrinsic material absorption. It is worth noting that translational motion in the plane of view of the trapping objective can be demonstrated straightforwardly, and is not shown here.

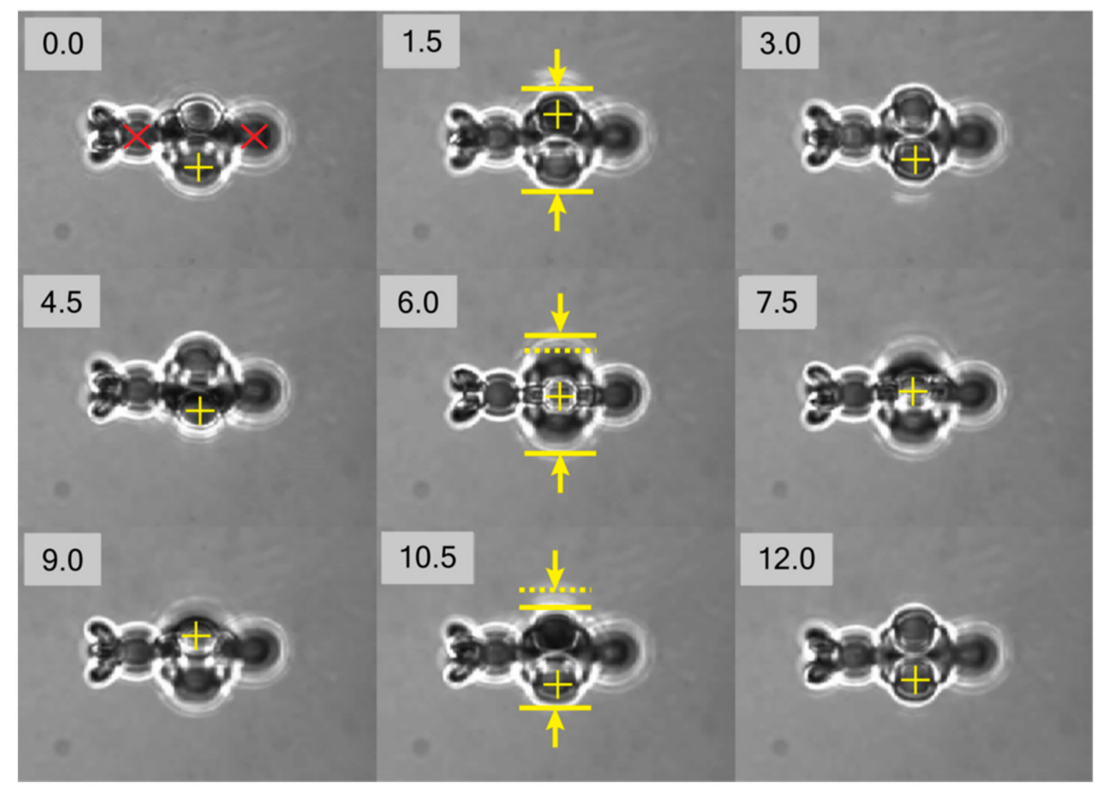

Figure 2. Frame sequence obtained from captured video demonstrating rotation of a trapped single micro-tool. Frames were extracted every $1.5 \mathrm{~s}$. Red crosses in frame at $0 \mathrm{~s}$ mark immobilization trap positions. Yellow crosses mark positions of displaced microspheres. The solid and dotted yellow lines shown in frames for 1.5, 6, and $10.5 \mathrm{~s}$ reveal variance of one of the dimensions of projection of the microtool captured by camera.

We analyze the rotation dynamics and trapping stability of individual micro-tool by image analysis algorithms. Sufficient contrast between the microtool and the background allows to implement edge detection algorithm. Each captured frame of the recorded video is processed as follows: the edges of the object are detected using a Sobel operator followed by the dilation and filling of gaps in resulting image, which allows to obtain binary mask corresponding to the object. The properties of the resulting binary images were analyzed using Matlab regionprops function. The center-of-mass (CoM) positions were extracted and axes of the equivalent ellipse with same normalized second moments as the original object binary image were obtained. These parameters were further used for analysis of motion of a single microtool.

The information on CoM position over each frame is presented as probability distribution in Figure 3a. The respective data on $X$ and $Y$ position distributions is presented in Figure 3b. The stiffness of trapping potential was analyzed using equipartition theorem and allowed to obtain values of $\mathbf{k}_{\mathbf{x}}=2.22 \mathrm{pN} / \mu \mathrm{m}$ and $\mathbf{k}_{\mathbf{y}}=1.96 \mathrm{pN} / \mu \mathrm{m}$. The values of the trapping potential stiffness could be used for assessment of the maximum possible force which could be exerted on the immobilized cell in order to assess individual cell stiffness [29].

The equivalent ellipse minor axis variance over time is presented in Figure 3c. The periodic variations of the value can clearly be observed, which can be attributed to rotation of the microtool. The corresponding Fourier spectrum of the extracted signal is presented in Figure $3 \mathrm{~d}$. Fourier analysis of the time-varying parameter allowed to reveal processes with different periodicity $-\mathrm{T}_{1}=8.6 \mathrm{~s}$ 
$(0.117 \mathrm{~Hz})$, which corresponds to complete 360 degrees revolution of the microtool and $\mathrm{T}_{2}=3.3 \mathrm{~s}$

$(0.3 \mathrm{~Hz})$ which could be attributed to out-of-plane rotation of the tool by $120^{\circ}$.
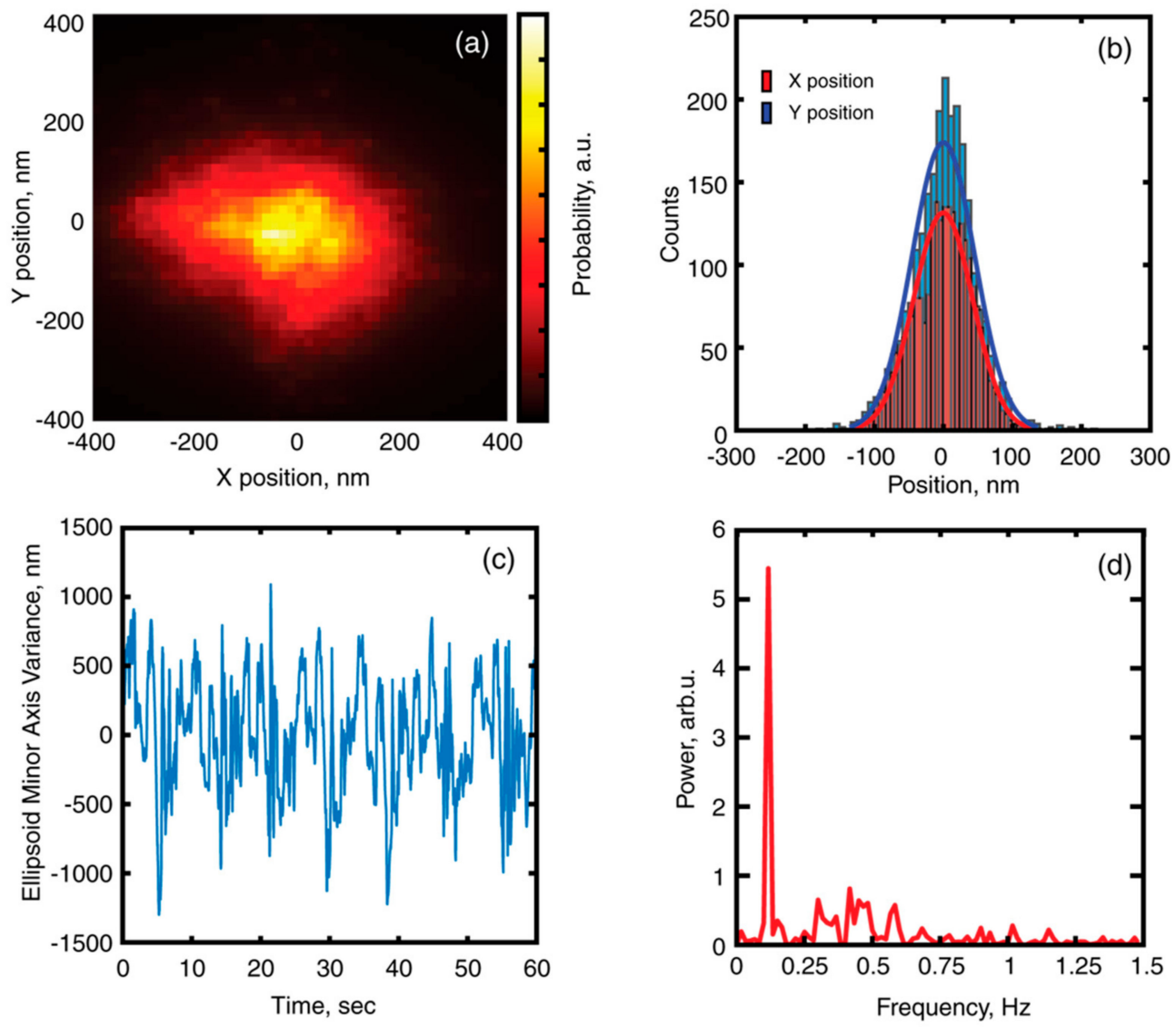

Figure 3. (a) Probability distribution of a center of mass (CoM) for single microtool. (b) Position distribution for $\mathrm{X}$ and $\mathrm{Y}$ coordinates of CoM. (c) Variance of equivalent ellipsoid minor axis, as a function of time (d) Power spectrum of the ellipsoid minor axis variance.

In order to demonstrate proof-of-concept, i.e., the capability of axial rotation of the living biological object, we have undertaken the experiments using yeast cells as the test object. For such an experiment, a pair of micro-tools were detached and immobilized in optical traps with relative separation of 15 microns between edges. After finding the object for studies, the tools were driven together to proximity (video is presented in Supplementary Materials Video S2), resulting in immobilization of the tested object. The success of the immobilization was checked by scanning with the microscope stage. The 'actuator' traps were turned on, resulting in simultaneous rotation of the trapped micro-tools, which transferred the torque on the object clamped between them. The frames of the captured video sequence are presented in Figure 4 (cell immobilization is shown in Supplementary Materials Video S3, cell rotation is shown in Supplementary Materials Video S4). 


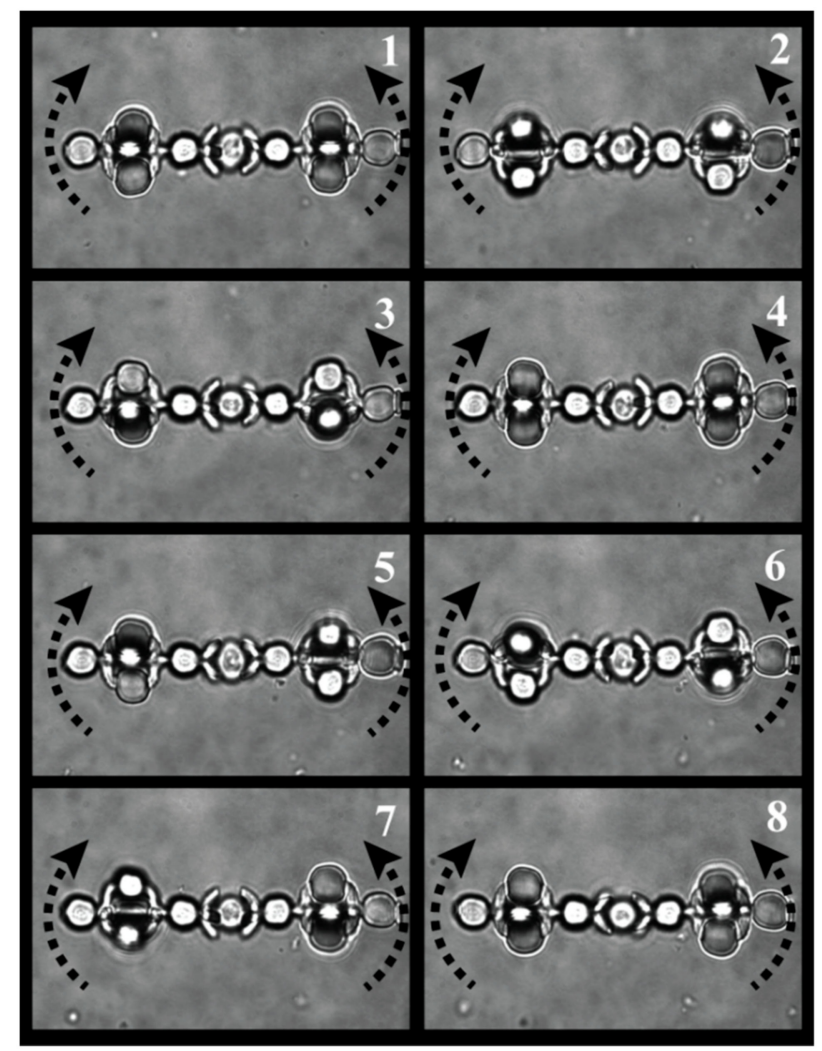

Figure 4. Frame sequence of rotation of trapped yeast cell. Frames were captured each second.

\section{Discussion}

The recorded videos and their frame-by-frame sequences demonstrate the feasibility of the proposed approach of using auxiliary structures for the micro-manipulation of objects. The analysis of motion of single microtool revealed the stability of microtool immobilization and out-of-plane rotation with the speed of 6-7 revolutions per minute was shown. The proof-of-principle manipulation of an individual live cell with a pair of microtools was demonstrated as well. However, several problems should be addressed in order to successfully implement these microtools for more complex studies.

First, for optical tomography applications, the rotation angle of the sample should be known. This can be achieved by the synchronization of rotation of the auxiliary tools. For this, one needs to project holograms with the out-of-plane position of 'actuator' trap in order to control the angle of revolution of the individual microtool. The current implementation of the trapping algorithm (e.g., static projection of 'actuator' trap) does not provide sufficient control over the rotation speed of the individual tool and does not synchronize the motion of a pair of tools.

Second, the Brownian motion of tools in liquid results in drifts of the studied object not only in the XY-plane, but in the Z-plane as well. The simple image processing technique implemented in this work allowed to determine the centroid position of a single microtool and can be further extended to the analysis of the motion of a pair of microtools with the cell immobilized in between them.

\section{Conclusions}

In conclusion, we presented a new approach that will allow a complete 360-degree scan of the biological object in-vitro embedded in its host fluid environment. For example, optical diffraction tomography (ODT) [30] allows for the measurement of the refractive index distribution of optically transparent objects, such as cancer cells (our proof of concept result appears in Figure 1d). The method does not require labeling or high intensity light sources. Crucially, the resolution of ODT depends on the range of the angle from which imaging takes place. Two-axis full rotation is optimal. Two principal 
approaches exist for the scan acquisition in ODT, namely illumination scanning [31] and sample scanning [32]. It should be noted that illumination scanning methods are constrained by limited projection angles [33]. Our proposed technique provides the possibility to performed such two-axis rotation by re-trapping a cell after one-axis rotation is performed. Experiments combining our technique and ODT are underway. These developments can open new horizons in microscopy, where accurate and full three-dimensional mapping of biological objects, and even other valuable functions can be performed with auxiliary optomechanically driven micro-tools.

Supplementary Materials: The following are available online at http://www.mdpi.com/2072-666X/11/1/90/s1, Video S1: 'Single fork rotation'. Video S2: 'Forks clamping'. Video S3: 'Forks cell trapping'. Video S4: 'Yeast rotation'.

Author Contributions: Conceptualization, P.G. and Y.R.; methodology, I.S., H.M. and Y.R.; software H.M. and Y.R.; formal analysis, I.S.; investigation, H.M.; resources, P.G. and Y.R.; data curation, H.M.; writing-original draft preparation, I.S.; writing-review and editing, P.G. and Y.R.; visualization, I.S.; supervision, P.G. and Y.R.; project administration, P.G.; funding acquisition, P.G. All authors have read and agreed to the published version of the manuscript.

Funding: This research was funded by ERC StG 'In Motion'.

Conflicts of Interest: The authors declare no conflict of interest.

\section{References}

1. Pawley, J.B. Handbook Of Biological Confocal Microscopy; Springer: Boston, MA, USA, 2006.

2. Denk, W.; Strickler, J.H.; Webb, W.W. Two-photon laser scanning fluorescence microscopy. Science 1990, 248, 73-76. [CrossRef]

3. Egner, A.; Hell, S.W. Fluorescence microscopy with super-resolved optical sections. Trends Cell Biol. 2005, 15, 207-215. [CrossRef]

4. Choi, W.J.; Park, K.S.; Eom, T.J.; Oh, M.-K.; Lee, B.H. Tomographic imaging of a suspending single live cell using optical tweezer-combined full-field optical coherence tomography. Opt. Lett. 2012, 37, 2784. [CrossRef] [PubMed]

5. Fauver, M.; Seibel, E.; Rahn, J.R.; Meyer, M.; Patten, F.; Neumann, T.; Nelson, A. Three-dimensional imaging of single isolated cell nuclei using optical projection tomography. Opt. Express 2005, 13, 4210-4223. [CrossRef] [PubMed]

6. Kus, A.; Dudek, M.; Kemper, B.; Kujawinska, M.; Vollmer, A. Tomographic phase microscopy of living three-dimensional cell cultures. J. Biomed. Opt. 2014, 19, 46009. [CrossRef] [PubMed]

7. Carmon, G.; Feingold, M. Rotation of single bacterial cells relative to the optical axis using optical tweezers. Opt. Lett. 2011, 36, 40. [CrossRef]

8. Tanaka, Y.; Wakida, S. Controlled 3D rotation of biological cells using optical multiple-force clamps. Biomed. Opt. Express 2014, 5, 2341. [CrossRef]

9. Habaza, M.; Gilboa, B.; Roichman, Y.; Shaked, N.T. Tomographic phase microscopy with $180^{\circ}$ rotation of live cells in suspension by holographic optical tweezers. Opt. Lett. 2015, 40,1881-1884. [CrossRef]

10. Kreysing, M.K.; Kießling, T.; Fritsch, A.; Dietrich, C.; Guck, J.R.; Käs, J.A. The optical cell rotator. Opt. Express 2008, 16, 16984. [CrossRef]

11. Kreysing, M.; Ott, D.; Schmidberger, M.J.; Otto, O.; Schürmann, M.; Martín-Badosa, E.; Whyte, G.; Guck, J. Dynamic operation of optical fibres beyond the single-mode regime facilitates the orientation of biological cells. Nat. Commun. 2014, 5, 5481. [CrossRef]

12. Müller, P.; Schürmann, M.; Chan, C.J.; Guck, J. Single-Cell Diffraction Tomography with Optofluidic Rotation about a Tilted Axis. In Proceedings of the SPIE 9548, Optical Trapping and Optical Micromanipulation XII, San Diego, CA, USA, 25 August 2015; p. 95480U.

13. Cao, B.; Kelbauskas, L.; Chan, S.; Shetty, R.M.; Smith, D.; Meldrum, D.R. Rotation of single live mammalian cells using dynamic holographic optical tweezers. Opt. Lasers Eng. 2017, 92, 70-75.

14. Phys, J.A. A microfluidic chip for single-cell 3D rotation enabling self-adaptive spatial localization A micro fl uidic chip for single-cell 3D rotation enabling self-adaptive spatial localization. J. Appl. Phys. 2019, 126, 234702. 
15. Liu, Y.; Cheng, D.K.; Sonek, G.J.; Berns, M.W.; Chapman, C.F.; Tromberg, B.J. Evidence for localized cell heating induced by infrared optical tweezers. Biophys. J. 1995, 68, 2137-2144. [CrossRef]

16. Mohanty, S.K.; Sharma, M.; Gupta, P.K. Generation of ROS in cells on exposure to CW and pulsed near-infrared laser tweezers. Photochem. Photobiol. Sci. 2006, 5, 134-139. [CrossRef] [PubMed]

17. Konig, K.; Liang, H.; Berns, M.W.; Tromberg, B.J. Cell damage in near-infrared multimode optical traps as a result of multiphoton absorption. Opt. Lett. 1996, 21, 1090-1092. [CrossRef] [PubMed]

18. Kawata, S.; Sun, H.B.; Tanaka, T.; Takada, K. Finer features for functional microdevices. Nature 2001, 412, 697-698. [CrossRef] [PubMed]

19. Goppert-Mayer, M. Uber Elementarakte mit zwei Quantensprungen. Ann. Phys. 1931, 401, $273-294$. [CrossRef]

20. Maruo, S.; Nakamura, O.; Kawata, S. Three-dimensional microfabrication with two-photon-absorbed photopolymerization. Opt. Lett. 1997, 22, 132-134. [CrossRef]

21. Phillips, D.B.; Simpson, S.H.; Grieve, J.A.; Bowman, R.; Gibson, G.M.; Padgett, M.J.; Rarity, J.G.; Hanna, S.; Miles, M.J.; Carberry, D.M. Force sensing with a shaped dielectric micro-tool. EPL 2012, 99, 58004. [CrossRef]

22. Gibson, G.M.; Bowman, R.W.; Linnenberger, A.; Dienerowitz, M.; Phillips, D.B. A compact holographic optical tweezers instrument. Rev. Sci. Instrum. 2012, 8, 113107. [CrossRef]

23. Phillips, D.B.; Padgett, M.J.; Hanna, S.; Ho, Y.L.D.; Carberry, D.M.; Miles, M.J.; Simpson, S.H. Shape-induced force fields in optical trapping. Nat. Photonics 2014, 8, 400-405. [CrossRef]

24. Villangca, M.J.; Palima, D.; Bañas, A.R.; Glückstad, J. Light-driven micro-tool equipped with a syringe function. Nat. Publ. Gr. 2016, 5, e16148-7. [CrossRef] [PubMed]

25. Palima, D.; Bañas, A.R.; Vizsnyiczai, G.; Kelemen, L.; Ormos, P.; Glückstad, J. Wave-guided optical waveguides. Opt. Express 2012, 20, 2004-2014. [CrossRef] [PubMed]

26. Asavei, T.; Nieminen, T.A.; Loke, V.L.Y.; Stilgoe, A.B.; Bowman, R.; Preece, D.; Padgett, M.J.; Heckenberg, N.R.; Rubinsztein-Dunlop, H. Optically trapped and driven paddle-wheel. New J. Phys. 2013, 15, 63016. [CrossRef]

27. Phillips, D.B.; Gibson, G.M.; Bowman, R.; Padgett, M.; Rarity, J.G.; Carberry, D.M.; Hanna, S.; Miles, M.J.; Simpson, S.H. Fashioning Microscopic Tools. In Proceedings of the Optics in the Life Sciences, Washington, DC, USA, 14-18 April 2013; p. TM2D.3.

28. Grier, D.G.; Roichman, Y. Holographic optical trapping. Appl. Opt. 2006, 45, 880-887. [CrossRef]

29. Guck, J.; Ananthakrishnan, R.; Mahmood, H.; Moon, T.J.; Cunningham, C.C.; Käs, J. The optical stretcher: A novel laser tool to micromanipulate cells. Biophys. J. 2001, 81, 767-784. [CrossRef]

30. Sung, Y.; Choi, W.; Fang-Yen, C.; Badizadegan, K.; Dasari, R.R.; Feld, M.S. Optical diffraction tomography for high resolution live cell imaging. Opt. Express 2009, 17, 266. [CrossRef]

31. Choi, W.; Fang-yen, C.; Badizadegan, K.; Oh, S.; Lue, N.; Dasari, R.R.; Feld, M.S. Tomographic phase microscopy. Nat. Methods 2007, 4, 717-719. [CrossRef]

32. Charrière, F.; Marian, A.; Montfort, F.; Kuehn, J.; Colomb, T.; Cuche, E.; Marquet, P.; Depeursinge, C. Cell refractive index tomography by digital holographic microscopy. Opt. Lett. 2006, 31, 178. [CrossRef]

33. Kawata, S.; Nakamura, O.; Minami, S. Optical microscope tomography I Support constraint. J. Opt. Soc. Am. A 1987, 4, 292. [CrossRef]

(C) 2020 by the authors. Licensee MDPI, Basel, Switzerland. This article is an open access article distributed under the terms and conditions of the Creative Commons Attribution (CC BY) license (http://creativecommons.org/licenses/by/4.0/). 